\title{
A role for the Fas antigen in lupus?
}

Although the immunopathogenesis of systemic lupus erythematosus remains poorly understood, the discovery in 1992 that underexpression of the apoptosis-encoding gene Fas accelerates autoimmune disease in the murine MRL/ lpr model of lupus, ${ }^{1}$ has taken the lupus community by storm. Numerous groups are currently examining the role of Fas in murine autoimmune disease. In addition, the recent observation that human patients with lupus may have elevated levels of a soluble Fas receptor, ${ }^{2}$ which could block Fas-mediated apoptosis of autoreactive lymphocytes, has further heightened the interest in the Fas molecule.

The Fas protein denotes the CD95 receptor and is a 45-48 $\mathrm{kD}$ type 1 membrane protein with sequence similarity to other members of a putative family of cell surface membrane receptors, including nerve growth factor receptor, CD40, and tumour necrosis factor receptor 1 (TNFR1). ${ }^{1,3}$ Nucleotide analysis of the cDNA encoding Fas and Apo-1 has shown that the Fas and Apo-1 genes are identical. The cytoplasmic domain of Fas, presumably essential for signal transduction, is most similar to CD40 and TNFR1. ${ }^{4}$ The Fas antigen is expressed on several lymphoma lines, EBV-transformed B lymphoblasts, activated B and T cells, ${ }^{3,5-7} \mathrm{CD} 4+\mathrm{CD} 8+$ thymocytes, $^{7}$ but not on resting or activated lymphocytes derived from MRL/lpr-lpr mice. ${ }^{7}$

A role for Fas in systemic lupus erythematosus pathogenesis is cogently demonstrated in the MRL/lpr-lpr model, a murine model of the human disease. ${ }^{8}$ In MRL/lpr mice, the presence of the Fas mutation (represented by the homozygous recessive 'lpr gene') results in an accelerated autoimmune syndrome characterised by high level IgG and autoantibody production, including high titers of antiDNA antibodies. ${ }^{8} \mathrm{MRL} / \mathrm{lpr}$ mice also develop arthritis, vasculitis, diseases of the central nervous system, skin disease, and an immune-complex mediated glomerulonephritis. ${ }^{9}$ With some notable exceptions, namely the presence of massive lymphadenopathy and expansion of a double negative $T$ cell population (CD4CD8-), the diversity of organ involvement and the immunopathologic characteristics in MRL/lpr mice resembles human lupus. ${ }^{9}$ The lpr mutation represents Fas underexpression, ${ }^{1}$ and essentially results from the integration of a $5.7 \mathrm{~kb}$ retroviral ETn retrotransposon sequence into the second intron of the Fas gene. Incorporation of this retrotransposon leads to defective transcription of the Fas gene and consequently to low-level expression of the Fas gene product. ${ }^{10,11}$ Recent studies have demonstrated that the Fas protein is expressed at high levels on CD4 + CD8 + thymocytes and activated mature lymphocytes in normal mice. ${ }^{7}$ In contrast, there is markedly defective expression of Fas by thymocytes and peripheral $\mathrm{T}$ cells derived from MRL/lpr mice which seems to protect these cells from apoptosis, ${ }^{12,13}$ and in so doing, has a profound effect on T-cell development. ${ }^{3,14}$ It is the protection of potentially autoreactive $\mathrm{T}$ cells from the apoptotic process that has been hypothesized to be the connection between autoimmunity and apoptosis. ${ }^{15}$ Indeed, the importance of the lpr mutation (Fas underexpression) in autoimmune pathogenesis has been underscored by a recent study demonstrating that the early correction of the lpr defect in $\mathrm{T}$ cells in transgenic MRL/lpr-lpr mice ameliorates both glomerulonephritis and lymphadenopathy as well as reducing autoantibody levels. ${ }^{16}$

Until recently, the revelance of $F a s$ to the aetiopathogenesis of the autoimmune syndrome in human lupus was unclear. However, recent data from Cheng and co-workers has generated potentially important insights into the role of Fas in autoimmune pathogenesis in human lupus. ${ }^{2}$ These investigators have recently reported that $60 \%$ of patients with lupus express an abnormal Fas transcript which codes for a soluble form of the Fas protein. ${ }^{2}$ This soluble Fas receptor protein appears to protect lymphocytes from apoptosis. Furthermore, the soluble form of Fas seems to be present at increased concentration in approximately $60 \%$ of patients with lupus. Cheng and co-workers have also demonstrated that the injection of a murine homologue to the human soluble Fas molecule results in abnormalities in both lymphocyte number and phenotype in normal laboratory mice. ${ }^{2}$ Thus, Cheng and colleagues observed an increase in both double negative $\mathrm{CD} 4-\mathrm{CD} 8$ - and single positive $\mathrm{CD} 4+$ or $\mathrm{CD} 8+\mathrm{T}$ cells, as well as a two to three-fold increase in both splenic $\mathrm{T}$ and $\mathrm{B}$ lymphocytes. Remarkably, the direct consequence of lymphocyte proliferation and phenotypic alterations was a four-fold increase in the response of syngenetic mixed cell culture proliferation by thymocytes and spleen cells from Fas-treated mice, compared to those from control-treated mice - a response suggestive of increased responsiveness to self-antigens.

Although the presence of increased Fas protein levels among patients with human lupus, as well as the induction of autoimmunity in eight-week-old CD1 mice, suggests that

\begin{tabular}{|l|}
\hline Apoptosis \\
\hline - physiologic programmed cell death \\
characterised by specific morphologic changes \\
(condensation of nuclear heterochromatin, cytoplasmic \\
shrinkage, cell shrinkage with retention of organelles, cell \\
membrane integrity is maintained for a prolonged period) \\
- active form of cell death (activate endonucleases, \\
macromolecular synthesis, etc) \\
- efficient removal of cells by phagocytosis
\end{tabular}

Ipr mutation/Fas underexpression:

- polyclonal hypergammaglobulinemia

- nephritis

- arthritis

- vasculitis

- CNS abnormalities

- skin lesions

- massive lymphadenopathy 


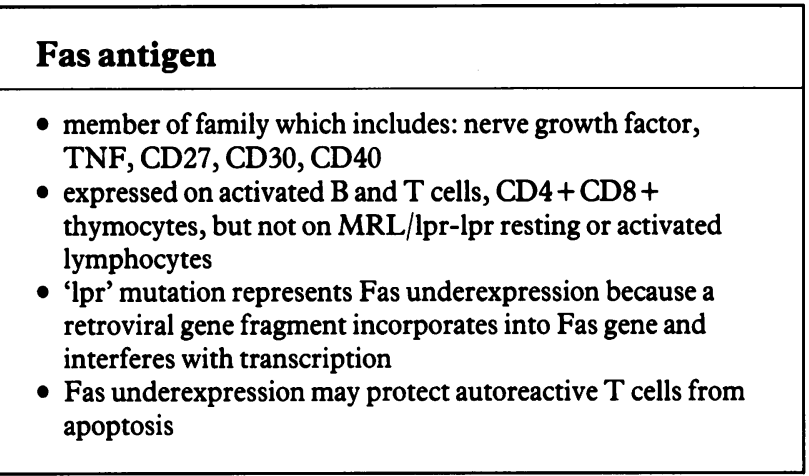

Box 3

the Fas molecule may play a critical role in the autoimmune syndrome in both lpr mice and human subjects with lupus, a recent study indicates that Fas expression may be normal in lupus patients. ${ }^{17}$ These investigators observed significantly higher levels of Fas expression on freshly isolated peripheral B cells and both $\mathrm{CD} 4+$ and $\mathrm{CD} 8+\mathrm{T}$ lymphocytes from lupus patients compared with normal controls. Moreover, no abnormalities in Fas regulation or in Fas-mediated apoptosis was detected in lupus B or T

1 Watanabe-Fukunaga R, Brannan CI, Copeland NG, Jenkins NA, Nagata S. Lymphoproliferation disorder in mice explained by defects in Fas antigen that mediates apoptosis. Nature 1992; 356: 314-7.

2 Cheng J, Zhou T, Liu C, et al. Protection from Fas-mediated apoptosis by a soluble form of the Fas molecule. Science 1994; 263: 1759-62.

3 Itoh N, Yonehara S, Ishii A, et al. The polypeptide encoded by the cDNA for human cell surface antigen Fas can mediate apoptosis. Cell 1991; 66: 233-43.

4 Yonehara S, Ishii A, Yonehara $M$. A cell-killing monoclonal antibody (anti-Fas) to a cell surface antigen co-downregulated with the receptor of tumor necrosis factor. $\mathcal{F}$ Exp Med 1989; 169: 1747-56.

5 Trauth BC, Klas C, Peters AM, et al. Monoclonal antibody-mediated tumor regression by induction of apoptosis. Science 1989; 245: 301-5.

6 Owen-Shaub LB, Meterissian S, Ford AJ. Fas/Apo-1 expression and function on malignant cells of hematologic and non-hematologic origin. $\mathcal{f}$ Immunother 1993; 14: 224-41.

7 Drappa J, Brot N, Elkon KB. The Fas protein is expressed at high levels on CD4 + CD8 + thymocytes and activated mature lymphocytes in normal mice but not in the lupus-prone strain, MRL/lpr. Proc Natl Acad Sci USA 1993; 90: 10340-4

8 Theofilopoulos AN, Dixon FJ. Murine models of systemic lupus erythematosus. Adv Immunol 1985; 37: 269-390.

9 Andrews BS, Eisenberg RS, Theofilopolous AN, et al. Spontaneous murine lupus-like syndromes. Clinical and immunopathological manifestations in several strains. ₹ Exp Med 1978; 148: 1198-215. lymphocytes compared to normal controls. Finally, no significant correlation between the percentage of cells expressing Fas and a clinical index of disease activity (lupus disease activity index, erythrocycte sedimentation rate, antinuclear antibodies, and anti-DNA antibody levels) could be discerned.

In conclusion, the elucidation of the underlying mechanisms leading to autoimmunity in lupus remains an elusive goal. Nonetheless, recent studies focusing on the Fas molecule have uncovered a critical role for the apoptosis-encoding Fas gene in mediating the autoimmune process. The intriguing possibility that the presence of a soluble form of Fas in the serum of lupus patients may protect autoreactive cells from apoptosis and thereby lead to autoimmunity provides an exciting connection between human lupus and a murine MRL/lpr model of the disease. With the emerging role of apoptosis in autoimmunity, another step appears to have been taken in unravelling the mystery of autoimmune pathogenesis in lupus.

AJAY K SINGH Division of Nephrology, Department of Medicine, New England Medical Centre and Tufts University School of Medicine, Boston, MA 02111, USA

Accepted 28 June 1995

10 Adachi $M$, Watanabe-Fukunaga $R$, Nagata $S$. Aberrant transcription caused by the insertion of an early transposable element in an intron of the Fas antigen gene of lpr mice. Proc Natl Acad Sci USA 1993; 90: 1756-60.

$11 \mathrm{Wu} \mathrm{J}$, Zhou T, He J, Mountz JD. Autoimmune disease in mice due to integration of an endogenous retrovirus in a apoptosis gene. $\mathcal{F}$ Exp Med 1993; 178: 461-8.

12 Ju ST, Cui H, Panka DJ, Ettinger A, Marshak-Rothstein A. Participation of target Fas protein in apoptosis pathway induced by CD4 + Th1 and CD8 + target Fas protein in apoptosis pathway induced by CD4+ Th1

13 Gillette-Ferguson I, Sidman CL. A specific intracellular pathway of apoptotic cell death is defective in the mature peripheral $\mathrm{T}$ cells of autoimmune lpr and gld mice. Eur $\mathcal{f}$ Immunol 1994; 24: 1181-5.

14 Andjelic S, Drappa J, Lacy E, Elkon KB, Nikolic-Zugic J. The onset of Fas expression parallels the acquisition of CD8 and CD4 in fetal and adult alpha beta thymocytes. Int Immunol 1994; 6: 73-9.

15 Mountz JD, Wu J, Cheng J, Zhou T. Autoimmune disease. A problem of defective apoptosis. Arthritis Rheum 1994; 37: 1415-20.

16 Wu J, Zhou T, Zhang J, He J, Gause WC, Mountz JD. Correction of accelerated autoimmune-disease by early replacement of the mutated lpr gene by the normal Fas apoptosis gene in the $T$ cells of transgenic MRL-lpr/lpr mice. Proc Natl Acad Sci USA 1994; 91: 2344-8.

17 Mysler E, Bini P, Drappa J, et al. The apoptosis-1/Fas protein in human systemic lupus erythematosus. 7 Clin Invest 1994; 93: 1029-34. 